ISSN: 2224-0616

Int. J . Agril. Res. Innov. \& Tech. 4 (2): 1-5, December, 2014

Available online at http:// www.ijarit.webs.com

\title{
EFFECT OF SEASONAL AND ENVIRONMENTAL VARIATION ON YIELD AND YIELD COMPONENTS OF HYBRID MAIZE
}

\author{
M.K. Islam1, M.S. Mahfuz²*, M.A.I. Sarker', S. Ghosh ${ }^{3}$ and A.S.M.Y. Ali ${ }^{3}$
}

Received 18 June 2014, Revised 20 October 2014, Accepted 22 December 2014, Published online 31 December 2014

\begin{abstract}
The experiment was conducted during kharif II in 2006 \& 07, rabi in 2006-07 \& 07-08 and kharif I in 2007 and 2008 at ARS, Burirhat, Rangpur in RCB design to understand the influence of season and location specific environment effect on growth and yield of hybrid maize and selected suitable variety(s). Four hybrid maize varieties $\mathrm{V}_{1}=\mathrm{BARI}$ hybrid Maize (BHM) - 2, $\mathrm{V}_{2}=\mathrm{BHM}-3, \mathrm{~V}_{3}=\mathrm{BHM}-5$ and $\mathrm{V}_{4}=$ Pacific-984 (as check) were tested during kharif II in 2006 while seven varieties $\mathrm{V}_{1}=\mathrm{BHM}-2, \mathrm{~V}_{2}=\mathrm{BHM}-3, \mathrm{~V}_{3}=\mathrm{BHM}-5$ and $\mathrm{V}_{4}=$ Pacific-984, $\mathrm{V}_{5}=$ Pacific- $60, \mathrm{~V}_{6}=$ Pacific- 11 and $\mathrm{V}_{7}=$ Prolin were during the other seasons except rabi, 0708, where variety Pacific- 555 was used in $V_{7}$ instead of Prolin. Seeds were sown on August 30 and August 11 for kharif II of 2006 and 2007, respectively, November 28 and 17 for rabi 2006-2007 and 2007-08 and March 08 and 12 for for kharif I 2007 and 2008. The crops were harvested on J anuary 25, 2007 and J anuary 12, 2008 in the two consecutive kharif II seasons; May 10 and April 29 in rabi 2006-07 and 2007-08, and July 01 and 05 in kharif I 2007 and 2008, respectively. Yield parameters were mostly varied significantly. The highest yield was obtained from BHM-5 (9.03 t ha-1), which was followed by Pacific-984 (8.89 tha1), BHM-3 (8.81 t ha-1) and BHM-2 (8.58 t ha-1) in kharif II, 2006 while in kharif II, 2007 the highest significant yield was noted in Pacific-984 (9.22 $\mathrm{t} \mathrm{ha}^{-1}$ ). In rabi, 2006-07, significant highest grain yield was obtained from Pacific-60 (11.03 t ha-1), which was statistically identical with Prolin (10.20 t ha-1). The yield of Prolin was also statistically identical with Pacific-11 (10.01 t ha-1), BHM-5 (10.00 t ha-1), BHM-3 (9.92 t ha-1) and BHM-2 (9.51 t ha-1). Comparatively lower temperature during ear initiation (mean $29.6^{\circ} \mathrm{C}$ in rabi and $31^{\circ} \mathrm{C}$ in kharif II) and silking (mean $18.2^{\circ} \mathrm{C}$ in rabi and $20.2^{\circ} \mathrm{C}$ in kharif II) contributed much for higher trend of yield in rabi over kharif. In kharif I, 2007, the highest yield (9.55 t ha-1) was recorded from Pacific-60, which was identical to Pacific-984 (9.25 t ha-1), BHM-5 (9.11 t ha1) and BHM-3 (8.89 $\left.\mathrm{t} \mathrm{ha}^{-1}\right)$. All the BARI hybrid maize varieties were suitable to grown in kharif I, kharif II and rabi season although BHM-3, BHM-5, Pacific-60 and Pacific-984 were better in Kharif I and Pacific-60, BHM-5, Prolin and Pacific-555 were found better in Rabi season.
\end{abstract}

Keywords: Season, Environment, Yield, Maize

\footnotetext{
${ }^{1}$ Senior Scientific Officer, Bangladesh Agricultural Research Institute, Burirhat, Rangpur, Bangladesh

${ }^{2}$ Regional Farm Broadcasting Officer, Agriculture Information Service, Rangpur, Bangladesh

${ }^{3}$ Scientific Officer, Bangladesh Agricultural Research Institute, Burirhat, Rangpur, Bangladesh

*Corresponding author's email: shohag_agri@yahoo.com (M.S. Mahfuz)
}

\section{Introduction}

Among the cereals maize ranks $3^{\text {rd }}$ position after rice and wheat. Maize can be grown throughout the year. High yield per hectare, high food value and multipurpose use as well as its high demand expanding maize cultivation day by day. There is a demand of 1.2 million tons of maize only to feed the poultry industries of Bangladesh (BARI, 2001). Again, for starch industries, other industries and for human consumption maize has a great demand. The yield of hybrid maize is about $20-30 \%$ higher than that of open pollination composite ones, which encouraged the farmers to prefer hybrid maize. Now, $87 \%$ of the total maize area is under hybrid maize. Recently BARI has developed 5 hybrid maize varieties. High quality protein maize (QPM) rich in tryptophen and lycin associated with recently evolved BARI hybrid maize-5 and other predominant BARI hybrid maize varieties along with other commercial hybrid maize varieties, the performance of the same across the seasons as well as across the locations is yet to be studied.

The environmental scientist working with maize have concluded from long term weather data that higher mean seasonal temperature was negatively correlated with grain yield and final grain yield was dependent on the rate and duration of grain growth and dry matter accumulation (BARI, 2001; Law and Brander, 1999). Increase in temperature decreased net photosynthesis and 
optimum temperature ranged from $28^{\circ} \mathrm{C}$ to $37.5^{\circ} \mathrm{C}$ for higher photosynthesis (Brandner and Salvucci, 2002). Physiological parameter like NAR depends on temperature due to enzyme activity rubisco and at $28^{\circ} \mathrm{C}$ rubisco oxygenase activity was 100\% (Edwards et al., 2001). However, the relationship among physiological parameters with the yield and yield components in three contrasting season has not been worked out in detail. The present investigation was carried out to understand the influence of season and location specific environment effect on growth and yield of hybrid maize and selected suitable variety (s) adaptive for specific areas.

\section{Materials and Methods}

The experiment was conducted during kharif II season of 2006 \& 07, rabi in 2006-07 \& 07-08, kharif I in 2007 at sandy loam soils of ARS, Burirhat, Rangpur following RCB design with 3 replications. The treatments included 4 hybrid maize varieties namely: $\mathrm{V}_{1}=\mathrm{BARI}$ hybrid maize (BHM) $-2, V_{2}=$ BHM-3, $V_{3}=$ BHM-5 and $V_{4}=$ Pacific-984 (as check) in kharif II, 2006 and seven varieties viz. $\mathrm{V}_{1}=\mathrm{BHM}-2, \mathrm{~V}_{2}=\mathrm{BHM}-3, \mathrm{~V}_{3}=$ BHM-5 and $V_{4}=$ Pacific- $984, V_{5}=$ Pacific- $60, V_{6}=$ Pacific- 11 and $V_{7}=$ Prolin in other seasons except rabi, 07-08 where variety Pacific-555 was used in $\mathrm{V}_{7}$ instead of Prolin. Seeds were treated with Vitavex 200 @ $5 \mathrm{~g} \mathrm{~kg}^{-1}$ seed for 10 minutes in airtight bag then kept open. Blanket application of cow dung @ $10 \mathrm{t} \mathrm{ha}^{-1}$ was made 7 days before sowing. The field was fertilized with 200-60-11045-5-2 kg NPKSZn and B/ha, respectively. Onethird amount of $\mathrm{N}$ as Urea and rest full amount of all other fertilizer were applied in experimental unit plot one day before sowing. Seeds of different hybrids maize varieties were accommodated in $4.5 \mathrm{~m} \times 6.0 \mathrm{~m}$ sized plot. Two seeds/ hill were sown on August 30 and August 11 for kharif II, 2006 and 2007, respectively, November 28 and 17 for rabi 2006-07 and 07-08, and March 08 and 12 for kharif I, 2007 and 2008 , at spacing $75 \mathrm{~cm} \times 20 \mathrm{~cm}$. Necessary gap filling were made by re-sowing within 8 days of sowing (DAS). Rest $2 / 3^{\text {rd }}$ Urea in equal splits was top dressed (TD) at 30 and 60 DAS. Weeding and mulching were done properly; final earthling up was made after $2^{\text {nd }} \mathrm{TD}$, which was followed by irrigation. Futher irrigation was made at 75 and 90 DAS. Despite that plant received about 405 $\mathrm{mm}$ and $554 \mathrm{~mm}$ of precipitation in kharif II 2006 and 2007 respectively. In rabi, the crop was irrigated 4 times at 60, 90, 110 and 120 DAS. The crop also received rainfall at $2^{\text {nd }}$ decades of December and February, slight in the month of March and 3rd decade of April in rabi, 2006-07. The crop in kharif I was irrigated 4 times at 10 days interval starting form 25 DAE. The crop also received a total of around $880 \mathrm{~mm}$ rainfall in Kharif I, 2007. Diazinon $60 \mathrm{EC} @ 2 \mathrm{ml} \mathrm{L}^{-1}$ of water was sprayed at 72 DAS. Guard was placed to avert bird (crow, parrot etc) damage. Minor variation about the maturity among the hybrid maize varieties was observed. The crops were harvested on January 25, 2007 and January 12, 2008 respectively for the two consecutive kharif II seasons of 2006 and 2007, May 10 and April 29 for rabi 2006-07 and 07-08, and July 01 and 05 for kharif I, 2007 and 2008.

Data on yield and yield components were taken properly, analyzed statistically and presented in tables 1 and 2 .

\section{Results and Discussion}

Traditionally hybrid maize is sown either rabi or kharif I season. Here the hybrid maize varieties were grown during kharif II season. In lieu of sowing in August 30 if the crop could be sown on mid J uly. The crop could be received about 600 $\mathrm{mm}$ rainfall and also assumed to be harvested within November 2006. Dowswell et al. (1996) also opined that, in most tropical environments, maize requires $600-700 \mathrm{~mm}$ of moisture well distributed over the growing season. Forty five per cent of total maize area in the developing tropical countries of the world covers 36.7 million hectares. The environment is characterized by a high mean temperature (around $28^{\circ} \mathrm{C}$ ) during the growing season (FAO, 1988). They have a high mean maximum temperature (around $32^{\circ} \mathrm{C}$ ) and a high mean minimum temperature (around $22^{\circ} \mathrm{C}$ ). At Rangpur region of Bangladesh there prevailed mean high temperature (around $3^{\circ} \mathrm{C}$ ) and high mean minimum temperature (around $26^{\circ} \mathrm{C}$ ) during growing season of maize. Sultan (2006) opined that the temperature $24^{0}$ to $30^{\circ} \mathrm{C}$ is suitable for maize production. He also opined that fluctuation of the temperature results in fluctuation in field duration of maize.

Table 1.a . Performance of hybrid maize varieties during kharif II season of 2006 season at ARS, Burirhat, Rangpur

\begin{tabular}{lcccccccccc}
\hline Treatments & $\begin{array}{c}\text { Plant } \\
\text { height } \\
(\mathrm{cm})\end{array}$ & $\begin{array}{c}\text { No. of } \\
\text { leaves/ } \\
\text { plant }\end{array}$ & $\begin{array}{c}\text { Days to } \\
50 \% \\
\text { tasseling }\end{array}$ & $\begin{array}{c}\text { Days to } \\
50 \% \\
\text { silking }\end{array}$ & $\begin{array}{c}\text { Ear } \\
\text { height } \\
(\mathrm{cm})\end{array}$ & $\begin{array}{c}\text { Cob } \\
\text { length } \\
(\mathrm{cm})\end{array}$ & $\begin{array}{c}\text { No. of } \\
\text { cobs/ } \\
\text { plant }\end{array}$ & $\begin{array}{c}\text { No. of } \\
\text { seeds/ } \\
\text { cob }\end{array}$ & $\begin{array}{c}1000 \\
\text { grain wt. } \\
(\mathrm{g})\end{array}$ & $\begin{array}{c}\text { Yield } \\
(\text { t ha-1) }\end{array}$ \\
\hline $\mathrm{V}_{1}=$ BHM-2 & $211 \mathrm{a}$ & 14 & $62 \mathrm{a}$ & $65 \mathrm{a}$ & $101 \mathrm{a}$ & $20.3 \mathrm{a}$ & $1.20 \mathrm{a}$ & $442 \mathrm{~b}$ & $325 \mathrm{a}$ & 8.58 \\
$\mathrm{~V}_{2}=$ BHM-3 & $216 \mathrm{a}$ & 13 & $61 \mathrm{a}$ & $64 \mathrm{a}$ & $96 \mathrm{a}$ & $19.7 \mathrm{a}$ & $1.10 \mathrm{~b}$ & $487 \mathrm{ab}$ & $318 \mathrm{a}$ & $8.81 \mathrm{~b}$ \\
$\mathrm{~V}_{3}=$ BHM-5 & $196 \mathrm{ab}$ & 12 & $55 \mathrm{~b}$ & $61 \mathrm{~b}$ & $80 \mathrm{~b}$ & $16.6 \mathrm{~b}$ & $1.29 \mathrm{a}$ & $474 \mathrm{~b}$ & $298 \mathrm{ab}$ & $9.03 \mathrm{a}$ \\
$\mathrm{V}_{4}=$ Pacific 984 & $186 \mathrm{~b}$ & 13 & $53 \mathrm{~b}$ & $57 \mathrm{~b}$ & $78 \mathrm{~b}$ & $19.7 \mathrm{a}$ & $1.02 \mathrm{~b}$ & $540 \mathrm{a}$ & $285 \mathrm{~b}$ & $8.89 \mathrm{~b}$ \\
\hline $\mathrm{CV}(\%)$ & 5.15 & 4.60 & 1.98 & 2.18 & 4.27 & 2.81 & 2.70 & 5.76 & 3.05 & 6.32 \\
\hline
\end{tabular}

Means in a column having similar or no letter did not differ significant at $5 \%$ level of significance 
Table 1.b. Performance of different hybrid maize during rabi season of 20006-07 at ARS, Burirhat, Rangpur

\begin{tabular}{|c|c|c|c|c|c|c|c|c|c|c|c|c|c|}
\hline \multirow[t]{2}{*}{ Treatment } & \multirow{2}{*}{$\begin{array}{l}\text { Plant } \\
\text { height } \\
\text { (cm) }\end{array}$} & \multirow{2}{*}{$\begin{array}{l}\text { No. of } \\
\text { leaves/ } \\
\text { plant }\end{array}$} & \multirow{2}{*}{$\begin{array}{c}\text { Days to } \\
50 \% \\
\text { tasseling }\end{array}$} & \multirow{2}{*}{$\begin{array}{c}\text { Days to } \\
50 \% \\
\text { silking }\end{array}$} & \multirow{2}{*}{$\begin{array}{l}\text { Ear } \\
\text { height } \\
\text { (cm) }\end{array}$} & \multirow{2}{*}{$\begin{array}{l}\text { No. of } \\
\text { cob/ } \\
\text { plant }\end{array}$} & \multicolumn{2}{|c|}{$\begin{array}{l}\text { Cob length } \\
\text { (cm) }\end{array}$} & \multicolumn{2}{|c|}{$\begin{array}{c}\text { No. of } \\
\text { grain/ cob }\end{array}$} & \multicolumn{2}{|c|}{$\begin{array}{c}1000 \text { grain } \\
\text { wt. (g) }\end{array}$} & \multirow[t]{2}{*}{$\begin{array}{l}\text { Yield } \\
\left(\text { ( ha- }^{-1}\right)\end{array}$} \\
\hline & & & & & & & Pri. & Sec. & Pri. & Sec. & Pri. & Sec. & \\
\hline $1=\mathrm{BHM}-2$ & $252 a$ & $16.7 \mathrm{a}$ & $109 a$ & $112 \mathrm{a}$ & $145 a$ & $1.29 \mathrm{ab}$ & $16.7 \mathrm{ab}$ & 10.5 & $384 d$ & $209 \mathrm{c}$ & 381ab & $331 b$ & 9.51b \\
\hline & & & & & $148 /$ & & & 9. & $430 c$ & $218 \mathrm{c}$ & $397 a$ & $322 \mathrm{bc}$ & $9.92 \mathrm{~b}$ \\
\hline & $223 b$ & & & $112 a$ & $130 \mathrm{~b}$ & & $16.2 \mathrm{~b}$ & 10.5 & 441bc & 258ab & $348 b$ & $285 c$ & $10.00 \mathrm{~b}$ \\
\hline fic-984 & 211b & & & 11 & $104 d$ & $1.03 d$ & 16.8ab & 10.0 & 474ab & 253b & 360ab & $290 \mathrm{bc}$ & $9.67 \mathrm{~b}$ \\
\hline & $250 a$ & & & & $136 b$ & & & 10. & 491a & 266ab & $375 a b$ & 372 & 11.03a \\
\hline C-11 & $224 b$ & 14 & 105 & 107 & $134 \mathrm{~b}$ & $1.21 \mathrm{bc}$ & $16.8 \mathrm{ab}$ & 10.3 & $417 \mathrm{~cd}$ & $280 a$ & $390 a$ & $303 \mathrm{bc}$ & $10.01 \mathrm{~b}$ \\
\hline & $225 b$ & 13.3d & $104 \mathrm{~b}$ & $106 \mathrm{~b}$ & $113 c$ & & $15.5 \mathrm{~b}$ & 8. & $01 \mathrm{a}$ & $198 c$ & $365 a b$ & 293bc & $10.20 \mathrm{ab}$ \\
\hline CV (\%) & 3.55 & 3.27 & 1.3 & .88 & 3.13 & 3.98 & 3.15 & 11.09 & 2.93 & 5.78 & 3.66 & 7.16 & 4.92 \\
\hline
\end{tabular}

Means in a column having similar or no letter did not differ significant at $5 \%$ level of significance

Table 1. Performance of different hybrid maize during kharif I season of 2007 at ARS, Burirhat, Rangpur

\begin{tabular}{|c|c|c|c|c|c|c|c|c|c|c|}
\hline Treatment & $\begin{array}{l}\text { Plant } \\
\text { height } \\
(\mathrm{cm})\end{array}$ & $\begin{array}{l}\text { No. of } \\
\text { leaves/ } \\
\text { plant }\end{array}$ & $\begin{array}{c}\text { Days to } \\
50 \% \\
\text { tasseling }\end{array}$ & $\begin{array}{c}\text { Days to } \\
50 \% \\
\text { silking }\end{array}$ & $\begin{array}{c}\text { Ear } \\
\text { height } \\
(\mathrm{cm})\end{array}$ & $\begin{array}{l}\text { No. of } \\
\text { cobs/ } \\
\text { Plant }\end{array}$ & $\begin{array}{l}\text { Cob } \\
\text { length } \\
\text { (cm) }\end{array}$ & $\begin{array}{l}\text { No. of } \\
\text { seeds/ } \\
\text { cob }\end{array}$ & $\begin{array}{c}1000 \\
\text { seeds wt. } \\
(\mathrm{g})\end{array}$ & $\begin{array}{c}\text { Yield } \\
\left(\mathrm{t} \mathrm{ha}^{-1}\right)\end{array}$ \\
\hline$\overline{\mathrm{V}_{1}}=\mathrm{BHM}-2$ & $245.07 a$ & $15.80 \mathrm{a}$ & $56.67 a$ & $58.67 a$ & $130.40 a$ & $1.08 \mathrm{ab}$ & 17.66 & $396 d$ & $328.00 a$ & $8.47 \mathrm{~b}$ \\
\hline $\mathrm{V}_{2}=\mathrm{BHM}-3$ & $237.20 a$ & $13.87 \mathrm{~b}$ & 55.67ab & 58.00ab & $107.80 \mathrm{~b}$ & 1.08ab & 18.84 & 430bcd & 308.70ab & 8.89ab \\
\hline $\mathrm{V}_{3}=\mathrm{BHM}-5$ & 200.87b & $12.47 \mathrm{bc}$ & 54.33bcd & 56.67abc & $84.00 \mathrm{bc}$ & $1.09 a$ & 17.36 & $460 \mathrm{bc}$ & 294.00b & 9.11ab \\
\hline $\mathrm{V}_{4}=$ Pacific -984 & 213.93b & $12.73 \mathrm{bc}$ & 53.33cd & $55.00 \mathrm{c}$ & 85.73bc & 1.05ab & 16.93 & $531 a$ & 295.00b & 9.25ab \\
\hline$V_{5}=$ Pacific -60 & $212.27 \mathrm{~b}$ & $12.87 \mathrm{bc}$ & $54.00 \mathrm{bcd}$ & $56.00 \mathrm{bc}$ & $90.60 \mathrm{bc}$ & 1.08ab & 16.88 & $476 \mathrm{~b}$ & $322.20 \mathrm{a}$ & $9.55 a$ \\
\hline $\mathrm{V}_{6}=$ Pacific -11 & 193.00b & $12.80 \mathrm{bc}$ & $53.00 \mathrm{~d}$ & 55.33c & $92.33 \mathrm{~b}$ & 1.07ab & 16.25 & $419 \mathrm{~cd}$ & 315.90ab & $8.54 \mathrm{~b}$ \\
\hline $\mathrm{V}_{7}=$ Prolin & 167.07C & $11.80 \mathrm{C}$ & 55.33abc & $58.00 \mathrm{ab}$ & 66.67c & $1.02 \mathrm{~b}$ & 16.97 & 425bcd & 306.90ab & $8.49 \mathrm{~b}$ \\
\hline CV (\%) & 4.06 & 5.05 & 2.19 & 2.39 & 9.62 & 2.16 & 8.07 & 4.49 & 3.95 & 4.64 \\
\hline
\end{tabular}

Means in a column having similar or no letter did not differ significant at $5 \%$ level of significance

All the parameters varied significantly in kharif II season except number of leaves/plant and yield in 2006-07 and days to 50\% silking in 2007-08. The highest grain yield $(9.03 \mathrm{t}$ ha-1) was associated with BARI hybrid maize-5 in 2006-07 while in 2007-08, the highest significant yield was noted in Pacific-984 (9.22 t ha-1), which was statistically similar with other varieties except

Table 2.a. Performance of different hybrid maize during kharif II season of 2007 at ARS, Burirhat, Rangpur

\begin{tabular}{lcccccccccc}
\hline Treatment & $\begin{array}{c}\text { Plant } \\
\text { height } \\
(\mathrm{cm})\end{array}$ & $\begin{array}{c}\text { No. of } \\
\text { leaves/ } \\
\text { plant }\end{array}$ & $\begin{array}{c}\text { Days to } \\
\text { 50\% } \\
\text { tasseling }\end{array}$ & $\begin{array}{c}\text { Days to } \\
\text { silking }\end{array}$ & $\begin{array}{c}\text { Ear } \\
\text { height } \\
(\mathrm{cm})\end{array}$ & $\begin{array}{c}\text { No. of } \\
\text { cobs/ } \\
\text { Plant }\end{array}$ & $\begin{array}{c}\text { Cob } \\
\text { length } \\
(\mathrm{cm})\end{array}$ & $\begin{array}{c}\text { No. of } \\
\text { seeds/ } \\
\text { cob }\end{array}$ & $\begin{array}{c}1000 \\
\text { seeds wt. } \\
(\mathrm{g})\end{array}$ & $\begin{array}{c}\text { Yield } \\
\left(\mathrm{t} \mathrm{ha}^{-1}\right)\end{array}$ \\
\hline $\mathrm{V}_{1}=$ BHM-2 & $248.80 \mathrm{a}$ & $15.87 \mathrm{a}$ & $60.33 \mathrm{abc}$ & 62.33 & $126.93 \mathrm{a}$ & $1.08 \mathrm{ab}$ & $18.60 \mathrm{a}$ & $421.00 \mathrm{c}$ & $321.67 \mathrm{a}$ & $7.47 \mathrm{~b}$ \\
$\mathrm{~V}_{2}=$ BHM-3 & $249.60 \mathrm{a}$ & $14.33 \mathrm{bc}$ & $61.67 \mathrm{ab}$ & 63.67 & $134.00 \mathrm{a}$ & $1.11 \mathrm{a}$ & $18.44 \mathrm{a}$ & $450.67 \mathrm{bc}$ & $323.00 \mathrm{a}$ & $8.30 \mathrm{ab}$ \\
$\mathrm{V}_{3}=$ BHM-5 & $238.47 \mathrm{ab}$ & $14.80 \mathrm{~b}$ & $62.67 \mathrm{a}$ & 63.67 & $129.73 \mathrm{c}$ & $1.10 \mathrm{ab}$ & $17.95 \mathrm{ab}$ & $440.67 \mathrm{c} 310.00 \mathrm{ab}$ & $9.01 \mathrm{a}$ \\
$\mathrm{V}_{4}=$ Pacific-984 & $215.20 \mathrm{~b}$ & $13.20 \mathrm{de}$ & $60.00 \mathrm{abc}$ & 62.33 & $101.73 \mathrm{c}$ & $1.02 \mathrm{c}$ & $18.63 \mathrm{a}$ & $531.33 \mathrm{a}$ & $311.00 \mathrm{ab}$ & $9.22 \mathrm{a}$ \\
$\mathrm{V}_{5}=$ Pacific-60 & $234.33 \mathrm{ab}$ & $14.00 \mathrm{bcd}$ & $58.00 \mathrm{c}$ & 61.00 & $123.07 \mathrm{ab}$ & $1.04 \mathrm{bc}$ & $16.29 \mathrm{bc}$ & $476.00 \mathrm{~b}$ & $312.00 \mathrm{ab}$ & $9.08 \mathrm{a}$ \\
$\mathrm{V}_{6}=$ Pacific-11 & $217.80 \mathrm{~b}$ & $13.53 \mathrm{cde}$ & $57.33 \mathrm{c}$ & 60.00 & $124.40 \mathrm{ab}$ & $1.05 \mathrm{bc}$ & $16.22 \mathrm{bc}$ & $419.33 \mathrm{c}$ & $300.00 \mathrm{~b}$ & $8.04 \mathrm{ab}$ \\
$\mathrm{V}_{7}=$ Prolin & $216.77 \mathrm{~b}$ & $12.80 \mathrm{e}$ & $59.00 \mathrm{bc}$ & 61.67 & $107.67 \mathrm{bc}$ & $1.01 \mathrm{c}$ & $14.60 \mathrm{c}$ & $427.00 \mathrm{c}$ & $298.00 \mathrm{~b}$ & $7.57 \mathrm{~b}$ \\
\hline \multicolumn{1}{c}{ CV $(\%)$} & 3.85 & 2.98 & 3.03 & 3.60 & 5.66 & 3.50 & 4.27 & 2.70 & 3.09 & 6.02 \\
\hline
\end{tabular}

Means in a column having similar or no letter did not differ significant at $5 \%$ level of significance

Table 2.b. Performance of different hybrid maize during rabi season of 2007-08 at ARS, Burirhat, Rangpur

\begin{tabular}{lcccccccccc}
\hline Treatment & $\begin{array}{c}\text { Plant } \\
\text { height } \\
(\mathrm{cm})\end{array}$ & $\begin{array}{c}\text { No. of } \\
\text { leaves/ } \\
\text { plant }\end{array}$ & $\begin{array}{c}\text { Days to } \\
50 \%\end{array}$ & $\begin{array}{c}\text { Days to } \\
\text { tasseling } \\
\text { silking }\end{array}$ & $\begin{array}{c}\text { Ear } \\
\text { height } \\
(\mathrm{cm})\end{array}$ & $\begin{array}{c}\text { No. of } \\
\text { cobs/ } \\
\text { Plant }\end{array}$ & $\begin{array}{c}\text { Cob } \\
\text { length } \\
(\mathrm{cm})\end{array}$ & $\begin{array}{c}\text { No. of } \\
\text { seeds/ } \\
\text { cob }\end{array}$ & $\begin{array}{c}1000 \text { seeds } \\
\text { wt. }(\mathrm{g})\end{array}$ & $\begin{array}{c}\text { Yield } \\
\left(\mathrm{t} \mathrm{ha}^{-1}\right)\end{array}$ \\
\hline $\mathrm{T}_{1}=$ BHM-2 & 203.33 & 14.57 & 106.00 & 108.00 & 104.53 & 1.13 & $16.78 \mathrm{ab}$ & $423.00 \mathrm{a}$ & $370.00 \mathrm{ab}$ & $9.53 \mathrm{~b}$ \\
$\mathrm{~T}_{2}=$ BHM-3 & 218.00 & 14.87 & 104.00 & 106.00 & 113.33 & 1.17 & $16.20 \mathrm{bc}$ & $415.00 \mathrm{a}$ & $343.33 \mathrm{c}$ & $9.70 \mathrm{~b}$ \\
$\mathrm{~T}_{3}=$ BHM-5 & 186.80 & 14.30 & 104.00 & 106.00 & 99.07 & 1.10 & $17.72 \mathrm{a}$ & $414.33 \mathrm{a}$ & $371.67 \mathrm{ab}$ & $11.08 \mathrm{a}$ \\
$\mathrm{T}_{4}=$ Pacific-984 & 205.53 & 14.57 & 103.33 & 105.33 & 102.93 & 1.20 & $16.15 \mathrm{bc}$ & $363.33 \mathrm{~b}$ & $351.67 \mathrm{bc}$ & $9.72 \mathrm{~b}$ \\
$\mathrm{~T}_{5}=$ Pacific-60 & 212.80 & 14.60 & 103.33 & 106.00 & 107.80 & 1.30 & $16.02 \mathrm{bc}$ & $386.00 \mathrm{ab}$ & $380.00 \mathrm{a}$ & $11.10 \mathrm{a}$ \\
$\mathrm{T}_{6}=$ Pacific-11 & 204.73 & 15.20 & 104.33 & 106.33 & 107.67 & 1.13 & $15.54 \mathrm{c}$ & $356.67 \mathrm{~b}$ & $370.00 \mathrm{ab}$ & $9.88 \mathrm{~b}$ \\
$\mathrm{~T}_{7}=$ Prolin & 219.07 & 15.03 & 103.67 & 106.00 & 115.60 & 1.13 & $16.11 \mathrm{bc}$ & $391.67 \mathrm{ab}$ & $360.00 \mathrm{abc}$ & $10.00 \mathrm{ab}$ \\
\hline $\mathrm{CV}(\%)$ & 5.18 & 3.84 & 2.12 & 3.14 & 9.50 & 8.57 & 3.45 & 4.52 & 3.60 & 6.03 \\
\hline
\end{tabular}

Means in a column having similar or no letter did not differ significant at $5 \%$ level of significance 
Table 2.c. Performance of different hybrid maize during kharif I season of 2008 at ARS, Burirhat, Rangpur

\begin{tabular}{lcccccccccc}
\hline Treatment & $\begin{array}{c}\text { Plant } \\
\text { height } \\
(\mathrm{cm})\end{array}$ & $\begin{array}{c}\text { No. of } \\
\text { leaves/ } \\
\text { plant }\end{array}$ & $\begin{array}{c}\text { Days to } \\
50 \% \\
\text { tasseling }\end{array}$ & $\begin{array}{c}\text { Days to } \\
\text { silking }\end{array}$ & $\begin{array}{c}\text { Ear } \\
\text { height } \\
(\mathrm{cm})\end{array}$ & $\begin{array}{c}\text { No. of } \\
\text { cobs/ } \\
\text { Plant }\end{array}$ & $\begin{array}{c}\text { Cob } \\
\text { length } \\
(\mathrm{cm})\end{array}$ & $\begin{array}{c}\text { No. of } \\
\text { seeds/ } \\
\text { cob }\end{array}$ & $\begin{array}{c}1000 \\
\text { seeds wt. } \\
(\mathrm{g})\end{array}$ & $\begin{array}{c}\text { Yield } \\
(\text { t ha-1) }\end{array}$ \\
\hline $\mathrm{T}_{1}=$ BHM-2 & $230 \mathrm{a}$ & $14.93 \mathrm{a}$ & $56.16 \mathrm{a}$ & $58.30 \mathrm{ab}$ & $120 \mathrm{a}$ & $1.12 \mathrm{a}$ & 16.5 & $370 \mathrm{~b}$ & $330 \mathrm{a}$ & $7.8 \mathrm{~b}$ \\
$\mathrm{~T}_{2}=$ BHM-3 & $229 \mathrm{a}$ & $14.75 \mathrm{a}$ & $55.9 \mathrm{a}$ & $59.03 \mathrm{a}$ & $110 \mathrm{ab}$ & $1.04 \mathrm{~b}$ & 17.0 & $375 \mathrm{a}$ & $310 \mathrm{~b}$ & $8.6 \mathrm{ab}$ \\
$\mathrm{T}_{3}=$ BHM-5 & $220 \mathrm{ab}$ & $14.30 \mathrm{bc}$ & $55.2 \mathrm{~b}$ & $57.10 \mathrm{~b}$ & $101 \mathrm{~b}$ & $1.08 \mathrm{ab}$ & 16.8 & $415 \mathrm{a}$ & $305 \mathrm{~b}$ & $9.0 \mathrm{a}$ \\
$\mathrm{T}_{4}=$ Pacific-984 & $225 \mathrm{a}$ & $14.70 \mathrm{ab}$ & $55.3 \mathrm{ab}$ & $57.06 \mathrm{bc}$ & $95 \mathrm{~b}$ & $1.10 \mathrm{a}$ & 16.7 & $400 \mathrm{ab}$ & $320 \mathrm{ab}$ & $8.7 \mathrm{ab}$ \\
$\mathrm{T}_{5}=$ Pacific-60 & $210 \mathrm{~b}$ & $14.50 \mathrm{~b}$ & $53.05 \mathrm{c}$ & $55.48 \mathrm{c}$ & $92 \mathrm{~b}$ & $1.09 \mathrm{ab}$ & 16.7 & $398 \mathrm{ab}$ & $340 \mathrm{a}$ & $9.4 \mathrm{a}$ \\
$\mathrm{T}_{6}=$ Pacific-11 & $217 \mathrm{~b}$ & $13.86 \mathrm{c}$ & $56.52 \mathrm{a}$ & $58.75 \mathrm{a}$ & $125 \mathrm{a}$ & $1.03 \mathrm{~b}$ & 16.4 & $372 \mathrm{~b}$ & $308 \mathrm{~b}$ & $8.3 \mathrm{ab}$ \\
$\mathrm{T}_{7}=$ Pacific-555 & $215 \mathrm{~b}$ & $14.60 \mathrm{~b}$ & $53.80 \mathrm{c}$ & $55.67 \mathrm{bc}$ & $98 \mathrm{~b}$ & $1.10 \mathrm{a}$ & 17.1 & $380 \mathrm{ab}$ & $320 \mathrm{ab}$ & $9.1 \mathrm{a}$ \\
\hline $\mathrm{LSD}(0.05)$ & 10.88 & 18.41 & 2.23 & 0.56 & 12.85 & - & - & - & - & - \\
\hline $\mathrm{CV}(\%)$ & 2.77 & 3.8 & 2.36 & 3.05 & 6.4 & 3.18 & 4.62 & 5.03 & 3.70 & 4.8 \\
\hline
\end{tabular}

Means in a column having similar or no letter did not differ significant at $5 \%$ level of significance

Table 1.b revealed that, in rabi 2006-07, the yield and all the yield-contributing characters except secondary cob length were significantly varied. Significant highest cob length $(17.9 \mathrm{~cm})$ along with significant highest number of grains/primary cob (491), higher number of grains/secondary cob (266), significant higher 1000 grain wt. of primary cob (375 g) and highest weight $(372 \mathrm{~g})$ of secondary cob contributed much to pacific-60 for giving significantly highest yield (11.03 t ha-1) which was statically identical to Prolin (10.20 t ha-1). The yield of Prolin was also statistically identical with other hybrid maize. In rabi, 2007-08, cob length, number of seeds/cob, 1000 seed weight and yield were varied significantly (Table 2.b). Pacific-60 was

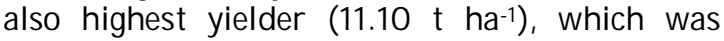
identically followed by BHM-5 (11.08 $\mathrm{t} \mathrm{ha}^{-1}$ ) and Pacific-555 (10.00 t ha-1) but significantly higher than the other varieties. Cumulative favourable effects of higher number of seeds/cob 386, 414.33 \& 391.67 and higher 1000 seed weight 380, $371.67 \& 360$, respectively contributed much for higher yields in Pacific-60, BHM-5 and Pacific555. The yield trend with hybrid maize during rabi season was comparatively higher than that of kharif II maize. This might be due to (about 40 $\mathrm{cm}$ ) taller plant stand associated with rabi maize. Again, average 2 more number of leaves/plant was observed in rabi maize than kharif II. Longer period taken for days to $50 \%$ tasseling (around 108 days) and days to $50 \%$ silking (around 111 days) in rabi, 2006-07 over kharif II maize (around 58 and 62 days, respectively). Comparatively lower temperature during ear initiation and silking of rabi maize (mean maximum around $29.6^{\circ} \mathrm{C}$ and mean minimum around $18.2^{\circ} \mathrm{C}$ ) over kharif II maize (mean maximum $31^{\circ} \mathrm{C}$ and mean minimum $20.2^{\circ} \mathrm{C}$ ) contributed much for higher trend of yield in rabi, 2006-07 over kharif II, 2007. Similar trend was also observed in the kharif II, 2008. BARI (2001) opined that increase in temperature decreased spikelet fertility and grain number was found to be dependent to temperature and radiation regimes during the period from ear initiation to silking.

In kharif I, all the parameters varied significantly except cob length in 2008. The highest yield in 2007 (9.55 t ha-1) was recorded from Pacific-60, which was identical to Pacific-984 (9.25 t ha-1), BHM-5 (9.11 t ha-1) and BHM-3 (8.89 t ha-1) but significantly higher than the rest varieties (Table1.c). Except Pacific-60, all other varieties produced identical yield. Significantly, higher number of cobs/plant (1.08) and 1000 grain weight $(322.20 \mathrm{~g})$ attributed to highest yield in Pacific-60. Similar trend was also observed in Pacific-984, BHM-5 and BHM-3 where either number of cobs per plant or number of seeds per plant alone or along with 1000-grain weight favoured the higher yield. Similar trend was observed in kharif I, 2008 where the highest yield (9.4 $\mathrm{t} \mathrm{ha}^{-1}$ ) was recorded from Pacific 60 identically followed by Pacific 555 (9.1 t ha-1), BHM 5 (9 t ha-1) BHM 3 (8.6 t ha-1) and Pacific 11 (8.3 t ha-1). BHM 2 produced significantly the lowest yield ( 7.8 t ha- $\left.^{-1}\right)$.

\section{Conclusion}

All the BARI hybrid maize varieties including check (Pacific-984) can be grown in kharif I and kharif II season although BHM-3, BHM-5, Pacific-60 and Pacific-984 are better in Kharif I. Again, considering the performance during rabi season, all the varieties can be grown as their yields were either higher or identical to the check variety Pacific-984 although Pacific-60, BHM-5, Prolin and Pacific-555 were found better.

\section{References}

BARI. 2001. CIMMYT sub-topical intermediate yellow-QPM hybird trail. Annual report of Bangladesh Agricultural Research Institute, Joydebpur, Gazzipur. pp. 44-67.

Brandner, S.J.C. and Salvucci, M.E. 2002. Sensivity of photosynthesis in a $\mathrm{C}_{4}$ plant, maize, to heat Stress. Plant Physiol. 129 (4): 1773-1780. 
Dowswell, C.R., Paliwal, R.L. and Cantrell, R.P 1996. Maize in third world. Winrock development oriented literature series. Steven A. Breth, series editor published in co operation with Winrock International Institute for Agricultural Development. p. 42.

Edwards, G.E, Furbank, R.T, Hatch, M.D. and Osmond, C.B. 2001. What does it takes to be $\mathrm{C}_{4}$ ? Lessons from the evolution of $\mathrm{C}_{4}$ photosynthesis. Plant Physiol. 125: 46-49.
FAO. 1988. FAO Production Year books, CIMMYT maize program. Food and Agricltural Organization, Rome, Italy.

Law, R.D. and Brandner, S.J.C. 1999. Inhibition and acclimation of photosynthesis to heat stress. Plant Physiol. 120: 173-181.

Sultan. 2006. Seed production technique of hybrid maize, Training Manual of "Hybrid Vhuttar Bis Utpadaner Kalakawshal". Bangladesh Agricultural Research Institute, Joydebpur, Gazipur. p. 21. 